\title{
Immunological effects of anti-coagulant unfractionated heparin in pregnant Chinese women with cryptic recurrent miscarriages
}

\author{
Yanli Hong ${ }^{1}$, Ke Hong ${ }^{2}$, Xiaojuan Chen ${ }^{3}$, Jinting Xue ${ }^{3}$, Yanyun Yin ${ }^{1}$
}

\author{
1Department of Reproductive Medicine, Affiliated Hospital of Nanjing University \\ of Chinese Medicine, Nanjing, Jiangsu, China \\ ${ }^{2}$ Department of Obstetrics and Gynaecology, Tongde Hospital of Zhejiang Province, \\ Hangzhou, Zhejiang, China \\ ${ }^{3}$ Gynaecology of Chinese Medicine, Nanjing University of Chinese Medicine, Nanjing, \\ Jiangsu, China
}

Submitted: 22 February 2020

Accepted: 11 April 2020

Arch Med Sci

DOI: https://doi.org/10.5114/aoms.2020.95054

Copyright $\odot 2020$ Termedia \& Banach

\begin{abstract}
Introduction: Anti-coagulant unfractionated heparin of low molecular weight (ACUHImw) therapy is popularly practised in the therapy of recurrent miscarriages (RMC) due to its anti-coagulant properties. However, several in vitro investigations have hypothesized about the possible immunological effects of ACUHImw.

Material and methods: We examined pregnant women with cryptic RMC (CRMC) to determine whether ACUHImw could regulate the immune reaction in vivo during their pregnancy. In this study, a total of 51 women were subjected to tinzaparin and 48 patients were considered as control women on the basis of an open single-centre randomized controlled trial. During different fertilization weeks (FW) 7, 19, 29, and 35 plasma samples were acquired for eleven chemokine and cytokine levels and then investigated by multiplex bead technology and selected to portray T-helper subset-related immunity. Results: We did not find any difference in chemokine C-C motif ligand-2, $-17,-22$, chemokine C-X-C motif ligand $-1,-8,-12,-13$ or cytokine interleukin- 6 when a mixed linear model test was carried out on ACUHImw in both the study and control women. However, differences were observed in the mixed linear model test on ACUHImw in both the study and control women during pregnancy of the Th1/Th17 related chemokine C-X-C motif ligand-10 $(p=0.01),-11(p<0.001)$ and chemokine C-C motif ligand-20 $(p=0.04)$ respectively.

Conclusions: A positive outcome of ACUHImw therapy in vivo was observed, thus establishing its potential proinflammatory effect. During $2^{\text {nd }}$ and $3^{\text {rd }}$ trimesters, the observed harmonious enlargement in Th1/Th17 related chemokine and cytokine levels does not recommend a fruitful immunological impact of ACUHImw therapy in vivo.
\end{abstract}

Key words: Th1/Th17, chemokine, cytokines, anti-coagulant unfractionated heparin, cryptic recurrent miscarriages.

\section{Introduction}

According to the earlier reported guidelines, practice of anti-coagulant unfractionated heparin of low molecular weight (ACUHImw) based therapy for women who suffered cryptic recurrent miscarriage (CRMC) is not

\author{
Corresponding author: \\ Yanli Hong \\ Department of Reproductive \\ Medicine \\ Affiliated Hospital of Nanjing \\ University of Chinese \\ Medicine \\ Nanjing, Jiangsu \\ China, 210000 \\ E-mail: \\ 15951711586@126.com
}


fruitful due to the data showing that its therapy does not lead to enhancement in live births. Still, ACUHImw therapy has been used in the medical field for the therapy of cRMC $[1,2]$. However, the reason behind this much less support for this therapy is the variations in the explanation of CRMC, disparity in examination patterns, haphazard therapy and screening methods in the available reports [3-7]. Recently heparin has been utilized in different types of cells due to its anti-coagulation properties in other medical fields $[8,9]$.

We know that various types of immune response are present in different subsets of T-helper (Th) cells in chemokines such as Th1-, Th2- and Th17-associated immunity [10-13]. Further, chemokines and cytokines act as markers of various types of immune effects because of their presence at assessable levels in plasma [14-16]. The chemokine C-X-C motif ligand-10 and -11 bind $C-X-C$ motif receptor-3 are interferon gamma induced chemokines and hence their role in engaging Th1 cells is very significant [11, $12,17]$. However, the cytokine interleukin-17 induced chemokines $\mathrm{C}-\mathrm{C}$ motif ligand-20 and -1 play a crucial role in enlistment of Th17 cells, showing C-C motif receptor- 6 as the receptor for chemokine C-C motif ligand-20 [16-18].

Chemokine C-C motif ligand-2 has an important part in $\mathrm{M} 2$-related fetal tolerance in pregnancy and Th2 cells are also activated and recruited by chemokine C-C motif ligand-2 [19]. In short, with the help of this study, we found that Th1/Th17 related immunity is unfavourable for a successful pregnancy while the enhancement of regulatory T cell and Th2 associated immunity is favourable for a successful pregnancy. Particularly, in the current era it is observed that enhancement of Th1/Th17 related immunity is associated with CRMC [20-22].

To the best of our knowledge this is the only report in which immunological effects of ACUHImw in pregnant Chinese women with cryptic recurrent miscarriages have been studied during pregnancy in vivo. Additionally, during the entire period of pregnancy plasma levels of cytokines and chemokines were measured in ACUHImw with respect to the study and control women.

\section{Material and methods}

\section{Study design}

Our investigation has been approved by our institutional review board. A study cohort of pregnant women with a history of CRMC was designed in such a way that those women were recruited between $1^{\text {st }}$ April, 2017 and $31^{\text {st }}$ December 2018 for our analysis. Only those participants who had a history of 4 or more instinctive successive abortions in the past before 23 weeks of fertilization (FW) and with an unknown cause of recurrent miscarriages were eligible to participate in this study. Another inclusion criterion was the history of CRMC in pregnant women as recommended previously by healthcare centre(s). For this study, we selected pregnant women only once their pregnancy was confirmed with the help of ultrasound. This study is totally based on their medical records and history of participating women and only those women were included in this study who were ready to participate and then inclusion and exclusion criteria were applied to them (Table I). First of all, according to national instructions all

Table I. Characteristic features of the participants of our study

\begin{tabular}{|c|c|c|c|}
\hline Characteristics ${ }^{\circledR}$ & Control $(n=48)$ & Study* $(n=51)$ & $P$-value \\
\hline Age & $32.6 \pm 4.1$ & $32.9 \pm 4.4$ & 0.91 \\
\hline Body mass index & $23.8 \pm 3.9$ & $24.7 \pm 4.1$ & 0.31 \\
\hline Pregnancy & $4.3 \pm 1.2$ & $4.9 \pm 1.9$ & 0.20 \\
\hline Miscarriage history & $3.4 \pm 1.1$ & $4.1 \pm 1.8$ & 0.27 \\
\hline RMC & 24 & 21 & 0.37 \\
\hline $\begin{array}{l}\text { Live births' history } \\
\text { (overall/1/2/3)\# }\end{array}$ & $(0.7 \pm 0.8) / 16 / 4 / 1$ & $(0.9 \pm 0.8) / 19 / 7 / 1$ & 0.41 \\
\hline Beginning FW & $7.1 \pm 1.8$ & $6.8 \pm 1.5$ & 0.49 \\
\hline Inclusion criteria & \multicolumn{3}{|c|}{$\begin{array}{l}\text { For more than or equal to eighteen years but less than forty-two years of age. } \\
\text { Informed consent from all the participating women. For more than or equal to three RMC. } \\
\text { If the reason of RMC was unknown. }\end{array}$} \\
\hline Exclusion criteria & \multicolumn{3}{|c|}{$\begin{array}{l}\text { If the reason of RMC was known. Any kind of infection/autoimmunity/kidney disease/ } \\
\text { neurological treatment diagnosed. Diabetic patients. Hyperthyroidism. Smoking habit. }\end{array}$} \\
\hline Discontinuity criteria & \multicolumn{3}{|c|}{$\begin{array}{l}\text { Any kind of severe complications raised after administration of tinzaparin was observed. } \\
\text { If the patient was found to have other anticoagulant protocol from any other institution. }\end{array}$} \\
\hline
\end{tabular}

- recurrent miscarriage. ${ }^{\# C h i l d ~ o r ~ c h i l d r e n . ~ F W-f e r t i l i z a t i o n ~ w e e k . ~}$ 
participating women underwent standardized diagnostic workup, along with the following: 1) gynaecological investigation, 2) sonohysterography, 3) parental karyotype, 4) board of endocrine evaluation, 5) vaginal ultrasonography, 6) hypercoagulability panel. The main aim of this study was to find out and verify the main source of RMC. Although all the aforementioned causes of RMC were eliminated, women were diagnosed with CRMC and only those women formed part of this study. At the time of inclusion, we acquired well-informed written consent of all the women and carefully cross-examined them. Before registration written consent was provided by all the patients.
In the beginning, the screening process was done with total of 147 women, but we randomly selected 99 women who further participated in this study. Out of 99 subjects, 51 women were considered as the study group and the remaining 48 were considered as the control group. In Table I, characteristic features of participating women are presented. During the study 8 women were eliminated from the study due to absence of consent to the study protocol and due to their critical health conditions (Table I, Figure 1). Hence only 91 women completed the study. Miscarriage occurred in 7 women and then 84 women continued their pregnancy above $23 \mathrm{FW}$ (Table II). Further, 6 more women were eliminated from the investigation

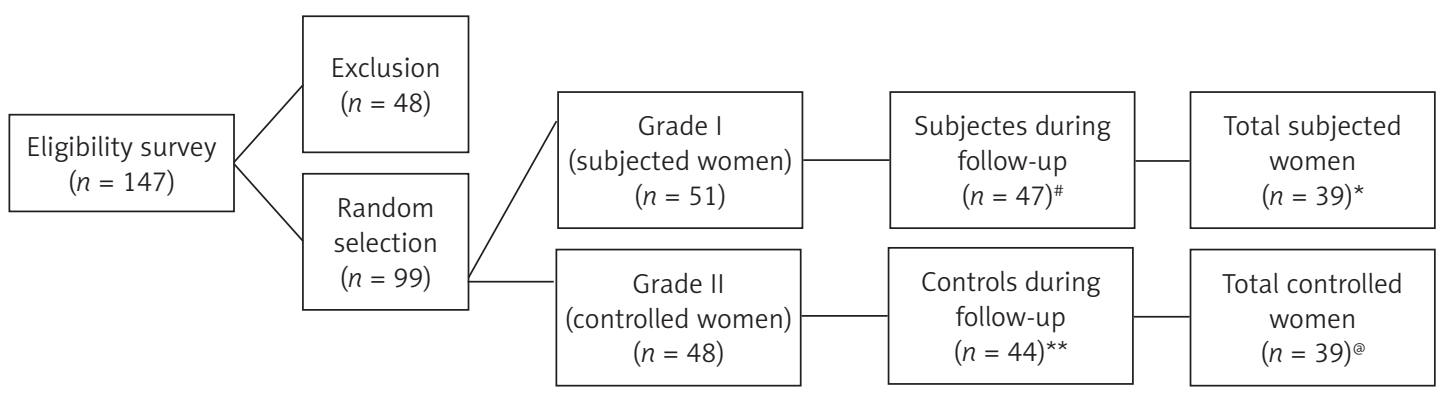

\#Due to discontinuity 4 women were excluded. *Out of 8 women, 4 were excluded due to missing samples and 4 were excluded due to miscarriage. **Total 4 women didn't turned up due their critical health conditions. @Out of 5 women, 2 were excluded due to missing samples and 3 were excluded due to miscarriage.

Figure 1. Hierarchy chart to represent the pregnant women's participation in our study

Table II. Outcome of pregnancy of the participants of our study

\begin{tabular}{|lccc|}
\hline Outcome of pregnancy & Control $(n=48)(\%)$ & Study ${ }^{\circledR}(n=51)(\%)$ & $P$-value \\
\hline Live births & $44(91.7)$ & $45(88.2)$ & 0.98 \\
\hline Normal birth & $34(70.8)$ & $27(52.9)$ & 0.21 \\
\hline Surgical birth & $8(16.7)$ & $14(27.4)$ & 0.24 \\
\hline Instrumental vaginal birth & $2(4.2)$ & $4(7.8)$ & 0.67 \\
\hline Full-term labour ${ }^{a}$ & $42(87.5)$ & $45(88.2)$ & 0.91 \\
\hline Pre-term labourb & $7(14.6)^{c}$ & $7(13.7)^{c}$ & 0.98 \\
\hline Preeclampsia/eclampsia\# & $1(2.1)$ & $2(3.9)$ & 0.98 \\
\hline Small-for-fertilization-age! & 0 & $3(5.9)$ & 0.19 \\
\hline Weight of infant at birth ${ }^{\text {c }}$ & $3511(529)$ & $3585(917)$ & 0.90 \\
\hline Fertilization diabetic & $1(2.1)$ & 0 & 0.51 \\
\hline Post-natal blood loss* & $2(4.2)$ & 0 & 0.19 \\
\hline Placental abruption & 0 & $1(2)$ & 0.98 \\
\hline Intrauterine fetal demise & $1(2.1)$ & $1(2)$ & 0.98 \\
\hline Stillbirth & $1(2.1)^{\mathrm{e}}$ & $5(9.8)$ & 0.98 \\
\hline RMC & $3(6.3)$ & 0 & 0.98 \\
\hline
\end{tabular}

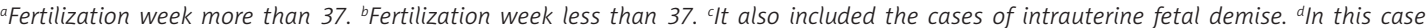
the values represent weight in grams (mean). elt included live birth. ${ }^{\circledR}$ To anti-coagulant unfractionated heparin of low molecular weight (ACUHImw) treatment. " and ' cases had different women participants. *More than $1 \mathrm{~L}$. RMC - recurrent miscarriage, FW-fertilization week. 
with respect to analyses of cytokines and chemokines due to loss of their blood samples.

\section{Drug administration}

A total of 99 qualified women were assigned to two groups, i.e. group 1 and group 2, after the confirmation of pregnancy, which was confirmed by ultrasound test. Group 1 (study group) of ACUHImw included 51 women who were subcutaneously injected with tinzaparin sodium (INNOHEP 4500 IU*; Guangzhou Pharmaceuticals, China) daily until FW 37. For group 2 (control group) women both active therapy and placebo were not applied in the selected 48 women.

All registered women were randomised through sequentially arranged sealed covers which were opened consecutively at the insertion end. In two different examination groups women were haphazardly designated according to the interference category noted on the sealed cover. After this point, no modification was incorporated in the allotment process. Possible immunological outcome of ACUHImw in vivo was referred as the main estimated consequence of this work. All scientific results were demonstrated including the terms of miscarriage rate, repetition of pregnancy obstacles, and/or any harmful impact of the medicine, used in the therapy, to the mother or to her infant.

\section{Sonographic analysis}

During this examination the national guidelines regarding antenatal care were pursued by the entire examination associates via a routine ultrasound test at FW 19-23, 9 postnatal weeks, during pregnancy midwife check-ups, blood sampling (PAPP-A, hCG) and supplementary chromosome $(13,18,21)$ test with combined ultrasound. Furthermore, at FW 11 and 13 levels transvaginal ultrasound screening was performed in all the women. According to national guidelines, at FW 19-23 level, some important test or scans were performed including routine ultrasound, cardiotocography (CTG), development assessment in 31 and 35 weeks, omphalic doppler assessment and cross examination at every level of scan for any hostile circumstance. In these examinations, women's antenatal data were entered on a data collection sheet including side effects of medicine, fetal viability and any other issues at each and every visit up to 14-21 days of miscarriage. In the above examination, if we did not find any abnormal value then there was no need to do further haematological check-ups.

\section{Plasma samples collection}

For this study, we collected blood samples of 99 women who had a past history of CRMC. To de- termine the immunological response of ACUHImw therapy in women with CRMC, we collected blood from them. During 4 different FW of pregnancy these samples were collected from the women, at the time of women's inclusion, without giving therapy to women (average FW 7, FW 19, FW 29 and FW 35) and after 14 days postnatally. Here we noted one more important thing about this sample collection process: that if a woman suffered from miscarriage after the first sample collection then we would not be able to collect a second sample for further investigation. Women who suffered miscarriages were not allowed to participate further in this study as to determine the response of ACUHImw during pregnancy it was important to analyse data in pairs.

Immunological assessment of anti-coagulant unfractionated heparin of low molecular weight study versus control women in vivo

We performed the measurements of cytokines and chemokines with multiplex bead assay using the Luminex 200 IS system (Millipore) installed with the MasterPlex QT 2010 software (MiraiBio). As per the protocol of the manufacturer (Millipore, Merck KGaA, Darmstadt, Germany), we used multiplex bead assay kits for immunological assessment of ACUHImw in vivo by analysing plasma samples with respect to the following analyses: chemokine C-C motif ligand-2, -17, -20, -22 and chemokine C-X-C motif ligand-1, -8, -10, -11, -12, -13 and cytokine interleukin- 6 with respective limit of detection values (all values are shown in pg/ $\mathrm{ml}$ ) as 15, 1.1, 9.2, 15.0, 15.0, 1.5, 15.0, 7.2. 3.7, 3.7, 1.5 (Table III). These analyses further represented the inflammation and immunity with respect to the following subsets of T helper (Th) cells: Th1 (chemokine C-X-C motif ligand-10 and -11), Th2 (chemokine C-X-C motif ligand-17 and -22), Th17 (chemokine C-X-C motif ligand- 1 and -8 and C-C motif ligand-20), as well as B cells (chemokine C-X-C motif ligand-13), general inflammation (cytokine interleukin-6, chemokine C-X-C motif ligand-8), monocyte recruitment and Th2/antiinflammatory (chemokine C-C motif ligand-2) and monocyte/T cell recruitment and Th2 (chemokine C-X-C motif ligand-12).

\section{Statistical analysis}

In earlier investigation it was observed that 82 women were enough for the detection of a $40 \%$ rise or reduction in immunological specification with $75 \%$ of capacity. According to the Kolmogorov-Smirnov test, we found that the data were not typically classified on the basis of chemokines and cytokines. To find out the variation in the chemokines and cytokines during pregnan- 


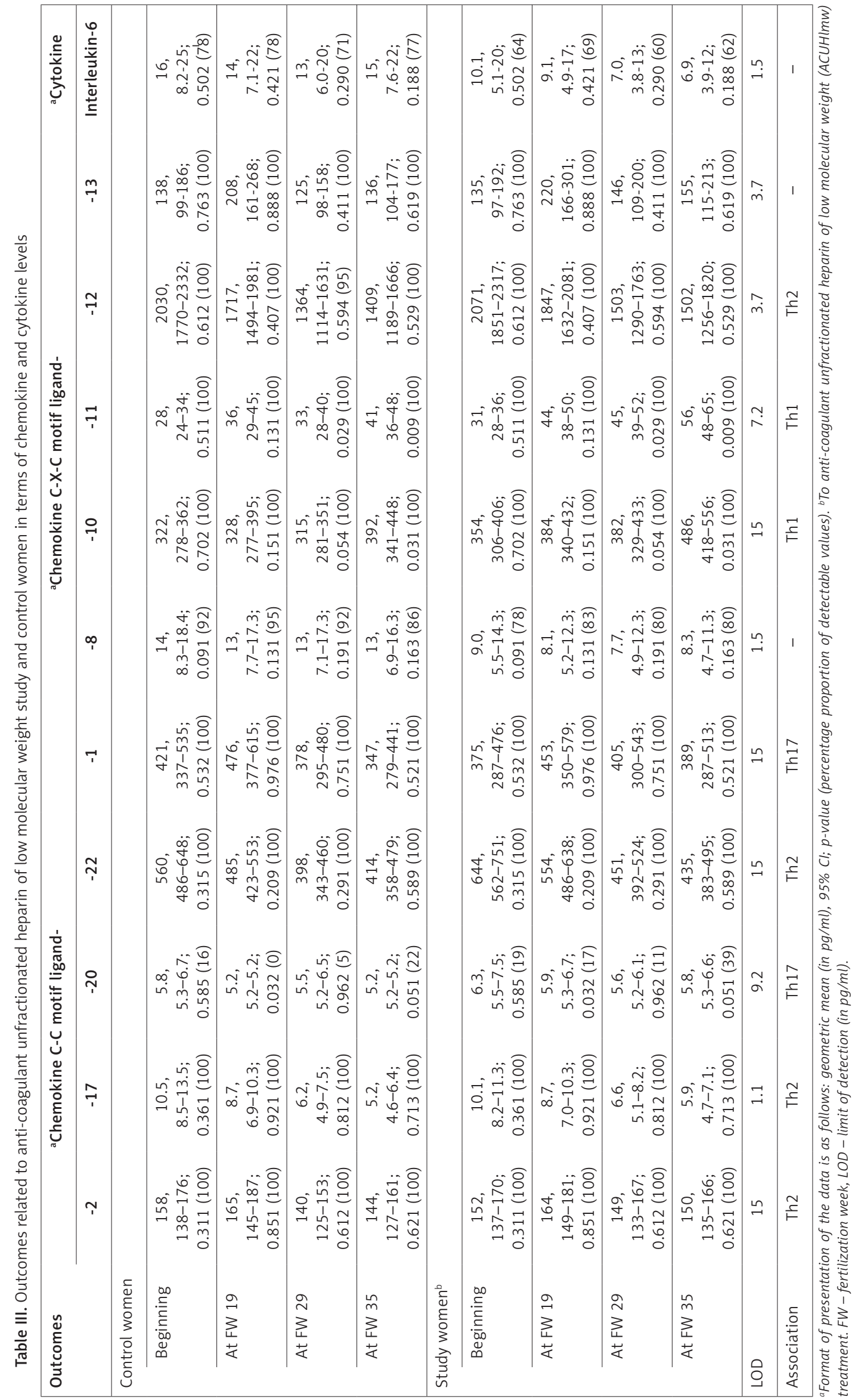


cy between both groups 1 and 2, a linear mixed model test was used. In this study a post-hoc test was preferentially performed (if $p<0.06$ ) in order to determine in which order and at which point of time there was any kind of variation. Due to the small percentage of samples with detectable levels, we used Fisher's exact test. During this study we found that in the linear mixed model test only 3 chemokines were greater than the value of 0.06 . Further, to find out the variation in chemokine and cytokine levels at inclusion and after 14 postnatal days, we used Student's t-test.

\section{Results}

\section{Study population characteristics}

In our study, total participating women numbered 99 and those were differentiated into two different groups: 51 women in the study group (Group 1) and 48 in the control group (Group 2) (Figure 1). In this study both the groups were congruent with respect to their number of earlier miscarriages, fertilization ages, body mass index and parity. Both study and control women had identical chance of miscarriage, $8.5 \%(4 / 47)$ and $6.8 \%(3 / 44)$ respectively. Thus, chemokines and cytokines were measured in 91 women but 8 women were excluded due to miscarriage and 6 women did not complete this study due to missing samples (Figure 1). For more consistent and decisive results chemokine levels were analysed and compared between women with a pregnancy more than FW $22(n=78)$ and women with an advanced miscarriage. Between the groups,

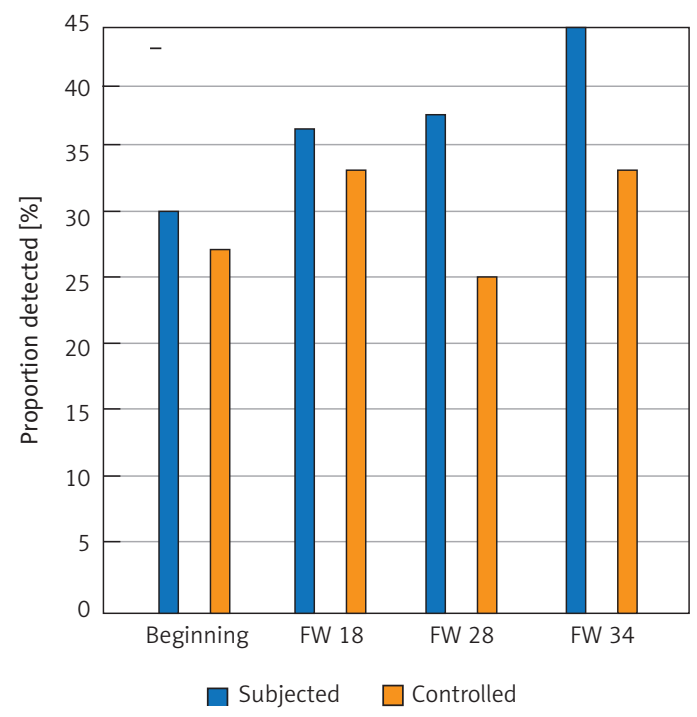

Figure 2. Comparison between study and control women with respect to chemokine C-C motif ligand-20 (in terms of \% proportion of women participants as per post-hoc test) we observed that only one chemokine, i.e. C-X-C motif ligand-11, was different (Student's $t$-test). During this study we observed that chemokine C-X-C motif ligand-11 was the single chemokine which showed higher levels at inclusion between the groups.

At FW 28 and FW 34 chemokine C-X-C motif ligand-10 and -11 levels were relevantly high in study women as compared to control women, which was further confirmed by unpaired Student's t-test. Later, a significant difference was also observed during the mixed model test regarding Th17 associated chemokine C-C motif ligand-20 between the study and control women (Figure 2). Since in many samples chemokine $\mathrm{C}-\mathrm{C}$ motif ligand-20 levels were found under the level of detection, for post hoc testing Fisher's exact test was applied and then we observed that Fisher's exact test showed more samples in study women with detectable levels in comparison to control women (0\% at both FW 19 and 35, in control women vs. $15 \%$ and $12 \%$ at FW 19 and 35 in study women). When we compared study and control groups with the help of linear mixed models, we did not observe any variation in the chemokine and cytokine levels from beginning to end of the pregnancy except for chemokine C-X-C motif ligand-10, -11 and chemokine C-C motif ligand-20 (Table III). Hence further post hoc testing was not required. In most of the samples investigated chemokine and cytokine were present at measurable levels with the exception of chemokine C-C motif ligand-20 (Table III). Between study and control women no significant difference was noted regarding chemokine and cytokine levels after 14 postnatal days.

\section{Gynaecological outcomes}

In this study, we found that both the study $(86 \%)$ and control (90\%) groups had very positive live birth rates. It was also observed that the premature birth rate was nominally high in women with CRMC history. In both groups the premature live birth rate was $12 \%$ and $13 \%$ respectively as compared to the Chinese population's live birth rate $(7.3 \%)$ [23]. In both the groups occurrence of postnatal bleeding, placental abruption and preeclampsia was found uniformly.

\section{Challenging outcomes}

During this study we did not find any severe maternal incident. Two control women were found with excessive bleeding at the time of childbirth and 4 study women and 3 control women were found with very limited vaginal bleeding. In the control group 1 gastroschisis and in study group 1 pleural effusion was observed with re- 
spect to fetal abnormality. In the control group no case of intrauterine growth restriction was found but in the study group 3 cases were found. On the other hand, in each group 1 intrauterine fetal death occurred and neonatal death occurred in 1 study woman. One of the ACUHImw therapy study women described soreness at the injection site. We did not find any ACUHImw therapy study women who had anti-coagulant unfractionated heparin induced thrombocytopenia. Women who were pregnant for more than FW 23 had a lower geometric mean $(25 \mathrm{pg} / \mathrm{ml})$ as compared to the women who were suffered from miscarriage (45 pg/ml; detailed data not shown).

\section{Discussion}

In this study our main aim was to determine the outcome of ACUHImw therapy in pregnant women with a history of CRMC in vivo. We found that chemokine C-X-C motif ligand-10 and -11 (Th1 related chemokines) and chemokine C-C motif ligand-20 (Th17 related chemokine) have a symbolic boost in plasma levels in the $2^{\text {nd }}$ and $3^{\text {rd }}$ phase of pregnancy in study women with ACUHImw as compared to those in control women. In this way, we may say that this study is the first of its kind to report the assessment of immunological effects of ACUHImw in vivo in women with a history of CRMC. The advantage of this study is that it is based on a randomized supervised longitudinal type sample study. Additionally, a proper board of markers was used in this study.

Furthermore, the clinical implication of our study is that as compared to control women, in ACUHImw study women increased plasma levels of the Th1 related chemokine $\mathrm{C}-\mathrm{C}$ motif ligand-10 and C-X-C motif ligand-11 were found in FW 29 and 35 . The relevance and importance of this result were supported by earlier reports which showed that interferon gamma induced both the Th1 related chemokines $\mathrm{C}-\mathrm{X}-\mathrm{C}$ motif ligand-10 and $-11[24,25]$. Another important thing about ACUHImw therapy is that it should be applied in the early stage of pregnancy for the best results due to early potential need of a Th1 response. With the help of this study one more important fact was observed: the women who had higher Th1 related chemokine C-X-C motif ligand-11 levels (just before the therapy) were more prone to miscarriage in comparison to the women whose pregnancy continued even after FW 23. In the case of Th17 related chemokine C-C motif ligand-20 the plasma levels were greater than the limit of detection at FW 19 and 35 in ACUHImw treated study women in comparison to the control women. This result is quite congruent with a similar kind of report in which non-pregnant subjects having a past history of CRMC had an enhanced level of Th17 associated chemokine C-C motif ligand-20 [26].

The limitation of this study is that during the analysis fetal-maternal interface environment was not expressed by the peripheral blood sample. However, another limitation was found, as our result with respect to the association between the potentially pro-inflammatory effect and Th-related effect of ACUHImw in vivo is quite contradictory to the previous result in which anti-inflammatory potential was observed [27-30]. Thus, we may say that we are unable to establish any outcome in this regard. The present examination did not cover the evaluation of markers of regulatory $T$ cells due to lack of an exclusive chemokine receptor profile.

In this examination our main aim was to investigate immunological effects of ACUHImw therapy and not to find out whether any kind of clinical implication is possible in pregnant Chinese women with CRMC or not. We also observed that during the $2^{\text {nd }}$ and $3^{\text {rd }}$ trimester of pregnancy immunological variations do not support the use of ACUHImw as an anti-inflammatory response. Our work can be further extended towards the investigation of the theory related to the use of ACUHImw-allied pro-inflammatory upshot to improve the RMC's implantation procedure.

In conclusion, with the help of this study we found a positive outcome of ACUHImw therapy in vivo and thus established its potential proinflammatory effect. However, during the $2^{\text {nd }}$ and $3^{\text {rd }}$ trimesters of pregnancy the harmonious enlargement in the Th1/Th17 related chemokine and cytokine levels recommended a hostile consequence in the form of a failed immunological impact of ACUHImw therapy.

\section{Acknowledgments}

This study was supported by Youth Science Foundation of The National Natural Science Foundation of China (81904237) and Natural Science Foundation of Jiangsu Province (SBK2015040021).

\section{Conflict of interest}

The authors declare no conflict of interest.

\section{References}

1. Areia AL, Fonseca E, Areia M, Moura P. Low-molecularweight heparin plus aspirin versus aspirin alone in pregnant women with hereditary thrombophilia to improve live birth rate: meta-analysis of randomized controlled trials. Arch Gynecol Obstet 2016; 293: 81-6.

2. ESHRE Guideline Group on RPL, Bender Atik R, Christiansen OB, Elson J, et al. ESHRE guideline: recurrent pregnancy loss. Hum Reprod Open 2018; 2018: hoy004.

3. Rasmark Roepke E, Hellgren M, Hjertberg R, et al. Treatment efficacy for idiopathic recurrent pregnancy loss - a systematic review and meta-analyses. Acta Obstet Gynecol Scand 2018; 97: 921-41. 
4. Blomqvist L, Hellgren M, Strandell A. Acetylsalicylic acid does not prevent first-trimester unexplained recurrent pregnancy loss: a randomized controlled trial. Acta Obstet Gynecol Scand 2018; 97: 1365-72.

5. Shaaban OM, Abbas AM, Zahran KM, Fathalla MM, Anan MA, Salman SA. Low-molecular-weight heparin for the treatment of unexplained recurrent miscarriage with negative antiphospholipid antibodies: a random ized controlled trial. Clin Appl Thromb 2017; 23: 567-72.

6. de Jong PG, Kaandorp S, Di Nisio M, Goddijn M, Middeldorp S. Aspirin and/or heparin for women with unexplained recurrent miscarriage with or without inherited thrombophilia. Cochrane Database Syst Rev 2014; 7: CD004734.

7. Mak A, Cheung MW, Cheak AA, Ho RC. Combination of heparin and aspirin is superior to aspirin alone in enhancing live births in patients with recurrent preg nancy loss and positive anti-phospholipid antibodies: a meta-analysis of randomized controlled trials and meta-regression. Rheumatology (Oxford) 2010; 49: 281-8.

8. Bai N, Hou D, Mao C, Cheng L, Li N, Xiao Ming Mao. MiR$376 c-3 p$ targets heparin-binding EGF-like growth factor (HBEGF) to inhibit proliferation and invasion in medullary thyroid carcinoma cells. Arch Med Sci. doi: https:// doi.org/10.5114/aoms.2019.85244.

9. Urbaniak J, Owczarek K, Miłoński J, Pietkiewicz P, Jałocha-Kaczka A, Olszewski J. Evaluation of selected parameters of the coagulation system during the perioperative period in patients undergoing endoscopic surgery of the paranasal sinuses. Arch Med Sci. doi: https://doi. org/10.5114/aoms.2017.72544.

10. Yeh KW, Chiu CY, Su KW, et al. High cord blood CCL22/ CXCL10 chemokine ratios precede allergic sensitization in early childhood. Oncotarget 2017; 8: 7384-90.

11. McCully ML, Kouzeli A, Moser B. Peripheral tissue chemokines: homeostatic control of immune surveillance T Cells. Trends Immunol 2018; 39: 734-47.

12. Campanati A, Orciani M, Consales V, et al. Characterization and profiling of immunomodulatory genes in resident mesenchymal stem cells reflect the Th1-Th17/Th2 imbalance of psoriasis. Arch Dermatol Res 2014; 306 915-20.

13. Annunziato F, Cosmi L, Liotta F, Maggi E, Romagnani S. Main features of human T helper 17 cells. Ann N Y Acad Sci 2013; 1284: 66-70.

14. Mor G, Aldo P, Alvero AB. The unique immunological and microbial aspects of pregnancy. Nat Rev Immunol 2017; 17: 469-82.

15. Hayglass KT. The quest for predictive immune biomarkers. Clin Exp Allergy 2011; 41: 165659.

16. Henningsson AJ, Tjernberg I, Malmvall BE, Forsberg P, Ernerudh J. Indications of Th1 and Th17 responses in cerebrospinal fluid from patients with Lyme neuroborreliosis: a large retrospective study. J Neuroinflammation 2011; 8: 36.

17. Luckheeram RV, Zhou R, Verma AD, Xia B. CD4+T cells: differentiation and functions. Clin Dev Immunol 2012; 2012: 925135

18. Shinoda K, Hirahara K, Nakayama T. Maintenance of pathogenic Th2 cells in allergic disorders. Allergol Int 2017; 66: 369-76.

19. Lindau R, Mehta RB, Lash GE, et al. Interleukin-34 is present at the fetal-maternal interface and induces immunoregulatory macrophages of a decidual phenotype in vitro. Hum Reprod 2018; 33: 588-99.

20. Saito S, Nakashima A, Shima T, Ito M. Th1/Th2/Th17 and regulatory $\mathrm{T}$-Cell paradigm in pregnancy. Am J Reprod Immunol 2010; 63: 601-10.
21. Figueiredo AS, Schumacher A. The T helper type 17/regulatory T cell paradigm in pregnancy. Immunology 2016 148: 13-21.

22. Robertson SA, Care AS, Moldenhauer LM. Regulatory T cells in embryo implantation and the immune response to pregnancy. J Clin Invest 2018; 128: 4224-35.

23. Chen $\mathrm{C}$, Zhang J, Xia $\mathrm{H}$, et al. Epidemiology of preterm birth in China in 2015 and 2016: a nationwide survey. Lancet 2018; 392: S73.

24. Griffith JW, Sokol CL, Luster AD. Chemokines and chemokine receptors: positioning cells for host defense and immunity. Annu Rev Immunol 2014; 32: 659-702.

25. Ekman AK, Sigurdardottir G, Carlstrom M, Kartul N, Jenmalm MC, Enerback C. Systemically elevated Th1-, Th2 and Th17-associated chemokines in psoriasis vulgaris before and after ultraviolet B treatment. Acta Derm Venereol 2013; 93: 525-31.

26. Lissauer D, Goodyear O, Khanum R, Moss PAH, Kilby MD. Profile of maternal CD4 T-cell effector function during normal pregnancy and in women with a history of recurrent miscarriage. Clin Sci (Lond) 2014; 126: 347-54.

27. Raghupathy R. Pregnancy: success and failure within the Th1/Th2/Th3 paradigm. Semin Immunol 2001; 13: 219-27.

28. Pomin VH, Mulloy B. Current structural biology of the heparin interactome. Curr Opin Struct Biol 2015; 34: 17-25.

29. Bruno V, Svensson-Arvelund J, Rubér M, et al. Effects of low molecular weight heparin on the polarization and cytokine profile of macrophages and $\mathrm{T}$ helper cells in vitro. Sci Rep 2018; 8: 4166.

30. Molvarec A, Szarka A, Walentin S, et al. Serum leptin levels in relation to circulating cytokines, chemokines, adhesion molecules and angiogenic factors in normal pregnancy and preeclampsia. Reprod Biol Endocrinol 2011; 9: 124. 